\title{
Yang-Mills Effective Action from QCD at Finite Chemical Potential
}

\author{
C. Villavicencio and E. S. Fraga \\ Instituto de Física, Universidade Federal do Rio de Janeiro, CP: 68528, Rio de Janeiro, RJ 21945-970, Brazil
}

Received on September 29, 2006

\begin{abstract}
We present a construction of an effective Yang-Mills action for QCD, from the expansion of the fermionic determinant in terms of powers of the chemical potential at high temperature for the case of massless quarks. We analyze this expansion in the perturbative region and find that it gives extra spurious information. We propose for the non-perturbative sector a simplified effective action which, in principle, contains only the relevant information.
\end{abstract}

Keywords: Effective Yang-Mills action; Perturbative QCD; Finite chemical potential

There has been an increasing interest in the last few years in the sign problem or phase problem in QCD. For finite chemical potential, the fermion determinant matrix is non-positive definite, so it is not possible to perform Monte-Carlo simulations $[1,2]$. The Glasgow method [3] and sampling techniques [4] have made great advances in the description of phase transitions on the lattice, considering a set of parameters near the transition line. However, the problem is still far from being solved.

There is special interest in the region of high temperature and low chemical potential, since it corresponds to the highenergy heavy-ion collision scenario. In this regime it is possible to expand the fermion determinant in powers of $\mu / T$ [57]. This kind of expansion is what we are interested in investigating. As one performs this expansion, some questions arise: what order is enough to cut the expansion? which is its range of validity? how to distinguish between real and complex terms? Differently from what is done in the previously mentioned papers, we keep the expanded series as a functional of the gauge fields, giving rise to an effective non-local YangMills action.

\section{BASIC IDEA OF THE EXPANSION}

For simplicity, consider one-flavor massless quarks. The QCD generating functional at finite chemical potential in euclidean space is defined as

$$
Z=\int \mathcal{D} G \operatorname{det}\left(-i \not D+i \mu \gamma_{4}\right) e^{-S_{\mathrm{YM}}[G]}
$$

where $G$ are the gluon fields, also present in the covariant derivative $D$, and $S_{\text {YM }}$ is the Yang-Mills action. We can expand the fermion determinant in powers of the chemical potential assuming that $\mu<\Lambda \sim T$ :

$$
\begin{array}{r}
\operatorname{det}\left(-i \not D+i \mu \gamma_{4}\right)=\operatorname{det}(-i \not D) \exp \left\{-\sum_{s=1}^{\infty} \frac{(i \mu)^{s}}{s} \int_{Y_{1} \ldots Y_{s}}\right. \\
\left.\operatorname{Tr} \gamma_{4} S\left(Y_{2}, Y_{1}\right) \gamma_{4} S\left(Y_{3}, Y_{2}\right) \ldots \gamma_{4} S\left(Y_{1}, Y_{n}\right)\right\},
\end{array}
$$

where $S\left(Y_{b}, Y_{a}\right)$ is the dressed fermion propagator, which can be expressed as a series in powers of the gauge field and the

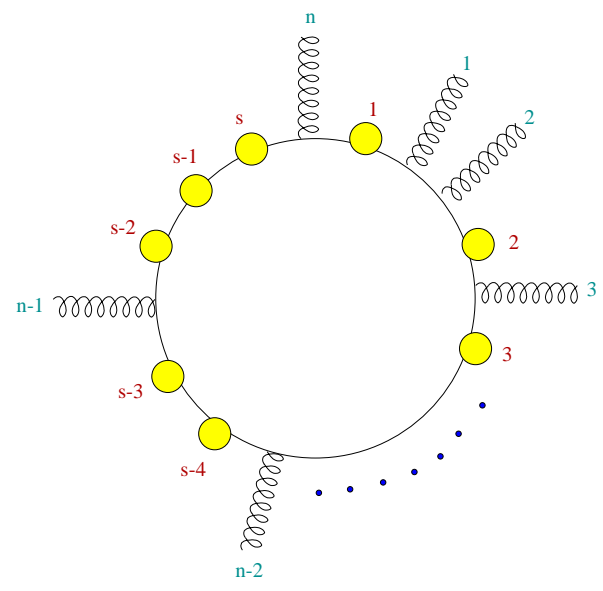

FIG. 1: General diagram for the construction of the effective vertices. The small circles correspond to chemical potential insertions.

free fermion propagator. The expansion, then, will contribute to additional terms in the Yang-Mills effective action

$$
S_{\mathrm{YM}}^{\mathrm{eff}}=S_{\mathrm{YM}}+\sum_{n=0}^{\infty} \int_{X_{1} \ldots X_{n}} \Gamma_{a_{1} \ldots a_{n}}^{(n)}{ }_{a_{1} \ldots \mu_{n}}^{\mu_{1}}\left(X_{i}\right) G_{\mu_{1}}^{a_{1}}\left(X_{1}\right) \ldots G_{\mu_{n}}^{a_{n}}\left(X_{n}\right),
$$

where the vertices $\Gamma^{(n)}$ are series in powers of the chemical potential: $\Gamma^{(n)}=\sum_{s \geq 1} \Gamma^{(n, s)}$, with $\Gamma^{(n, s)} \sim \mu^{s}$. In this way we have a positive-definite fermion determinant and the contribution from the chemical potential will be part of an effective Yang-Mills action. The problem now is to find criteria to cut this infinite series in terms of the chemical potential and gauge fields.

\section{FEYNMAN DIAGRAMS}

The Feynman rules to calculate the different effective vertices are almost the same as the usual ones in momentum space, except that all the operators between chemical potential insertions must be transposed in order. Figure 1 shows a general diagram with chemical potential and gluon insertions. The rules are:

- Draw the topologically different Feynman diagrams. Multiply by $-\gamma_{\mu_{i}} t_{a_{i}}$ for any gluon insertion, by $-i \mu \gamma_{4}$ 


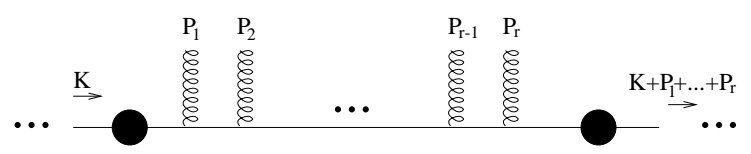

FIG. 2: Chemical potential insertions.

for any chemical potential insertion and divide by the symmetry factor $s$, the number of chemical potential insertions shown in (2).

- Between chemical potential insertions, the order of the operator must be transposed considering momentum conservation. An example is shown in Fig. 2.

This piece of the effective vertex integrand must be written as

$$
\begin{aligned}
& \left(-i \mu \gamma_{4}\right)\left[\tilde{S}_{F}(K)\left(-t_{a_{1}} \gamma_{\mu_{1}}\right) \tilde{S}_{F}\left(K+P_{1}\right) \ldots\right. \\
& \left.\ldots\left(-t_{a_{r}} \gamma_{\mu_{r}}\right) \tilde{S}_{F}\left(K+P_{1}+\cdots+P_{r}\right)\right]^{(t)}\left(-i \mu \gamma_{4}\right),
\end{aligned}
$$

where the exponent $(t)$ in the brackets indicate to transpose the order of the operators: $\left[O_{1} O_{2} \ldots O_{j-1} O_{j}\right]^{(t)}=$ $\mathrm{O}_{j} \mathrm{O}_{j-1} \ldots \mathrm{O}_{2} \mathrm{O}_{1}$.

- Take the trace over gamma matrices and color group representation, integrate over internal momentum and multiply by $(2 \pi)^{4} \delta\left(\sum P_{i}\right)$ (momentum conservation). The summation of the internal Matsubara frequencies are over Fermi-Dirac modes.

The sum of all diagrams will produce the effective vertices $\Gamma^{(n, s)}$ which will be invariant under any cyclic change in the set of indices $\mu_{i}, a_{i}, P_{i}$ (or $X_{i}$ in the case of configuration space). It is important that the effective vertices preserve explicitly this cyclic feature at the moment of expanding in the HTL approximation, although some diagrams are the same when contracted with the gluon fields in the action because they are invariant under interchange of any set of indices as can be seen in (3).

\section{PERTURBATIVE QCD}

To test the expansion, we study it firstly in the perturbative sector. Setting $G \rightarrow g G$, we find naturally the way to cut the gauge field series, which is given by the order of perturbative corrections. For one-loop corrections, we just need the effective action up to order $g^{2}$, so

$$
S_{\mathrm{YM}}^{\mathrm{eff}}=S_{\mathrm{YM}}+\Gamma^{(0)}+g^{2} \int_{X Y} \Gamma_{\mu \nu}^{(2)}{ }_{\mu \nu}^{a b}(X, Y) G_{\mu}^{a}(X) G_{v}^{b}(Y) .
$$

where this effective action is gauge invariant. If we calculate the pressure up to order $g^{2}$, considering the expansion on chemical potential up to order $\mu^{4}$, we surprisingly obtain the same result as in the usual perturbative QCD calculation with the chemical potential included in the quark propagator [8].
However, the next terms in the chemical potential series are non-zero. In particular, the next vacuum contribution for the pressure is

$$
P_{0}^{(6)}=-\frac{\Gamma^{(0,6)}}{\beta V} \approx 0.9334 N_{c} \frac{\mu^{6}}{\pi^{2} T^{2}},
$$

which is negligible only for $\mu \ll \Lambda$. More than establishing the range of validity, this result shows that this kind of expansion contains some spurious information, at least for the massless case, and in principle the only indication on where to cut the series is the dimension of the desired observable.

\section{NON-PERTURBATIVE QCD}

For the non-perturbative regime, we need another criterion to cut the series. The most appropriate is the Weinberg power counting [9], i.e. considering the external momentum as a small parameter, $p<\Lambda$, and also assuming that the chemical potential and the expectation value of the fields are of the same order, $\mu \sim G \sim p$. This gives a low-energy theory where the effective Lagrangian can be expanded in soft modes. In our case, we need that the chemical potential and the gluon field fluctuation be less than the external momentum. We are interested in not so low energies; if not, it is not possible to expand the fermion determinant. Since we want to see what will Weinberg criterion in this case is modified for not so low momentum as $\mu \sim G<p<\Lambda$.

In the case of Yang-Mills theories, this soft-mode expansions for high temperatures correspond to the Hard Thermal Loop (HTL) approximation [10, 11]. The minimal action must be of order $p^{4}$. So, applying the power counting, the minimal effective action is

$$
\begin{aligned}
S_{\mathrm{YM}}^{\min }[G, \mu]= & S_{\mathrm{YM}}[G]+S^{(0,2)}[\mu]+S^{(0,4)}[\mu] \\
& +S_{\mathrm{LO}}^{(2,2)}[G, \mu]+S_{\mathrm{LO}}^{(3,1)}[G, \mu],
\end{aligned}
$$

where the indices $(n, s)$ denote powers of $n$ in the gauge field and $s$ in the chemical potential, and LO means leading order in the HTL approximation. In this case, the appearance of imaginary terms will happen for $S_{\mathrm{LO}}^{(3,1)}$.

The whole series of gauge fields is gauge invariant in every order in the $\mu$ expansion, i.e. $\sum_{n} S^{(n, s)}$ is gauge invariant for all $s$ as can be seen directly from (2). The terms in the sum over chemical potential powers are traces containing dressed propagators, which are gauge invariant; so, every term in the sum is gauge invariant.

The minimal effective vertices we need satisfy the Ward identities $p \cdot \Gamma^{(2,2)}=0$ and $p \cdot \Gamma^{(3,1)}=0$. For the case of $\Gamma^{(2,2)}$ it was expected, since it basically reproduces the same result as that of performing radiative corrections to the gluon propagator with finite a density fermion propagator. For the case of $\Gamma^{(3,1)}$ it is not so obvious, since usually the contraction of the three gluon vertex with the external momentum gives a combination of the self-energy in the HTL approximation. However, there is no $\Gamma^{(2,1)}$, so necessarily, in a infinitesimal gauge shift $\delta G=\partial \alpha+[G, \alpha]$ on $\Gamma^{(3,1)}$, the contribution to $\Gamma^{(2,1)} \mathrm{com}$ ing from $\partial \alpha$ must give zero. HTL preserves gauge invariance 
by changing derivatives on the vertex operators to covariant derivatives.

The minimal vertices we consider with the power counting used will be functions of $p / p_{4}$ in the HTL approximation at leading order. It is easy to see because there are $\Gamma^{(2,2)} \mu^{2} G^{2}$ and $\Gamma^{(3,1)} \mu G^{3}$, both of dimension 4 , so the corresponding effective vertices, without the chemical potential dependence, are dimensionless. The next-to-leading-order terms will be powers of $p / T$. This allows us to make another approximation. Since $p_{4}=2 n \pi T$, then

$$
p / p_{4} \rightarrow\left\{\begin{array}{c}
\infty \text { for } n=0 \\
0 \text { for } n \neq 0
\end{array}\right.
$$

We can separate the fields then from the zero order of the Matsubara frequencies $G(X)=T G_{0}(\boldsymbol{x})+T \sum_{n \neq 0} e^{i 2 n \pi T x_{4}} G_{n}(\boldsymbol{x})$. giving an intermediate step before the Dimensional Reduction (DR) approximation [12-14]. .

This non-perturbative effective action is under construction and must be tested, but in principle it could provide rich infor- mation on the confinement-deconfinement transition line. The low-energy effective action proposed here as compared to the one obtained by DR [15] is that it allows for an analysis of lower temperatures, above and under where the region where the phase transition is supposed to happen. DR considers temperatures higher than the expected critical temperature.

The fact that contributions up to order $g^{2}$ in the expansion gives the same result for one-loop perturbative corrections to the pressure, and the next terms are non-zero, as can be seen in (5), indicates that next contributions are perturbations of the zero, and not necessarily will be negligible, so these effective actions are necessary in order to exclude such spurious information.

\section{Acknowledgment}

We acknowledge financial support from CAPES, CLAF, CNPq, FAPERJ and FUJB-UFRJ.
[1] S. Hands, Nucl. Phys. Proc. Suppl. 106, 142 (2002).

[2] E. Laermann and O. Philipsen, Ann. Rev. Nucl. Part. Sci. 53, $163(2003)$.

[3] I. M. Barbour and A. J. Bell, Nucl. Phys. B 372, 385 (1992).

[4] Z. Fodor and S. D. Katz, Phys. Lett. B 534, 87 (2002).

[5] S. A. Gottlieb, W. Liu, D. Toussaint, R. L. Renken, and R. L. Sugar, Phys. Rev. Lett. 59, 2247 (1987).

[6] C. R. Allton et al., Phys. Rev. D 68, 014507 (2003).

[7] C. R. Allton, W. Armour, D. Leinweber, A. Thomas, and R. Young, Phys. Rev. D 71, 054508 (2005).

[8] J. I. Kapusta, Finite Temperature Field Theory, Cambridge Uni- versity Press (1989).

[9] S. Weinberg, Physica A 96, 327 (1979).

[10] E. Braaten and R. D. Pisarski, Phys. Rev. D 45, 1827 (1992).

[11] J. Frenkel and J. C. Taylor, Nucl. Phys. B 374, 156 (1992).

[12] S. Weinberg, Phys. Lett. B 91, 51 (1980).

[13] P. H. Ginsparg, Nucl. Phys. B 170, 388 (1980).

[14] T. Appelquist and R. D. Pisarski, Phys. Rev. D 23, 2305 (1981).

[15] A. Hart, M. Laine, and O. Philipsen, Nucl. Phys. B 586, 443 (2000). 